\title{
Quantitative Magnetic Resonance Angiography in Internal Carotid Artery Occlusion with Primary Collateral Pathway
}

\author{
Yun Jung Bae, ${ }^{a}$ Cheolkyu Jung, ${ }^{a}$ Jae Hyoung Kim, ${ }^{a}$ Byung Se Choi, ${ }^{a}$ Eunhee Kim ${ }^{a, b}$ \\ ${ }^{a}$ Department of Radiology, Seoul National University College of Medicine, Seoul National University Bundang Hospital, Seongnam, Korea \\ bDepartment of Radiology, Hallym University Dongtan Sacred Heart Hospital, Hwaseong, Korea
}

Background and Purpose Quantitative magnetic resonance angiography (Q-MRA) enables direct measurement of volume flow rate (VFR) of intracranial arteries. We aimed to evaluate the collateral flows in internal carotid artery (ICA) occlusion with primary collateral pathway via circle of Willis using Q-MRA, and to compare them between patients who recently developed ipsilateral symptomatic ischemia and those who did not.

Methods Between 2012 and 2014, 505 patients underwent Q-MRA in our institution. Among these, 33 patients who had unilateral ICA occlusion with primary collateral pathway were identified, and grouped into asymptomatic patients, stable patients with chronic infarction, and symptomatic patients with acute/subacute infarction. Mean VFR (mVFR) in intracranial arteries was measured and compared between the patients' groups. Kruskal-Wallis test was used for statistical analysis.

Results Six patients were asymptomatic, fifteen with chronic infarction were stable, and twelve with acute/subacute infarction were symptomatic. The mVFR of ipsilateral middle cerebral artery in symptomatic patients was significantly lower than those in stable or asymptomatic patients $(73.7 \pm 45.6 \mathrm{~mL} / \mathrm{min}$ vs. $119.9 \pm 36.1 \mathrm{~mL} / \mathrm{min}$ vs. $121.8 \pm 42.0 \mathrm{~mL} / \mathrm{min}$; $P=0.04)$. Total sum of the mVFR of ipsilateral anterior, middle, and posterior cerebral arteries was significantly lower in symptomatic patients than those in other groups $(229.3 \pm 51.3$ $\mathrm{mL} / \mathrm{min}$ vs. $282.0 \pm 68.6 \mathrm{~mL} / \mathrm{min}$ vs. $314.0 \pm 44.4 \mathrm{~mL} / \mathrm{min} ; P=0.02$ ).

Conclusions Q-MRA could be helpful to demonstrate the difference in the degree of primary collateral flow in ICA occlusion between the patients with recent symptomatic ischemia and those without.

Keywords Magnetic resonance angiography; Carotid artery, internal; Collateral circulation
Correspondence: Cheolkyu Jung Department of Radiology, Seoul National University College of Medicine, Seoul National University Bundang Hospital, 82 Gumi-ro 173beon-gil, Bundang-gu, Seongnam 13620, Korea

Tel: +82-31-787-7623

Fax: +82-31-787-4011 E-mail: jck0097@gmail.com

Received: April 13, 2015 Revised: August 17, 2015 Accepted: August 25, 2015

This work was supported by Grant no. 11-2011-009 from the SNUBH Research Fund.

The authors have no financial conflicts of interest.

\section{Introduction}

Acute ischemic stroke by occlusion of internal carotid artery (ICA) is known to have relatively high rate of ischemic progression and recurrence. ${ }^{1}$ However, ICA occlusion can have variable clinical courses - from no symptom to severe neurologic deficit - according to the degree of collateral flow. ${ }^{2}$ Thus, the assessment of collateral flow in ICA occlusion is of great clinical significance.

The collateral circulation in ICA occlusion is constituted with primary collateral pathways via circle of Willis and secondary pathways via ophthalmic artery and leptomeningeal vessels. ${ }^{3}$ In particular, the presence of ipsilateral middle cerebral artery (MCA) flow via primary collateral pathway has been known to protect cerebral tissue from the progression of ischemic insult. 4,5 
Accordingly, the presence of MCA flow has proven that it may reduce initial stroke severity and be associated with early favorable outcome. $^{6}$

Recently, the advent of quantitative magnetic resonance angiography (Q-MRA) has enabled direct measurement of aforementioned intracranial flows. ${ }^{7,8}$ However, only a limited number of studies have quantitatively assessed the degree of collateral flow in ICA occlusion. Most recently, Rutgers et al. ${ }^{9}$ have measured quantitative volume flow via circle of Willis using Q-MRA, along with collateral flow via ophthalmic artery using transcranial Doppler, and that via leptomeningeal anastomosis using conventional angiography. However, the authors reached the conclusion that recurrent ischemic stroke with symptomatic carotid artery occlusion was associated with increased collateral flow through posterior communicating artery, which appeared to be conflicting finding against those from previous studies. ${ }^{5,6,10}$

In this study, we performed direct measurement of intracranial flow in unilateral ICA occlusion with primary Willisian collateral using Q-MRA. We used noninvasive optimal vascular analysis (NOVA; Vassol, Chicago, IL, USA) to calculate the mean volume flow rate ( $\mathrm{mVFR}$ ) of the intracranial arteries. The purpose of this study was to compare the mVFR of intracranial arteries between the symptomatic patients who recently developed cerebral ischemia ipsilateral to the occluded ICA and the stable or the asymptomatic patients.

\section{Methods}

\section{Subjects}

Between July, 2012 and September, 2014, 505 consecutive patients underwent Q-MRA in our institution as the diagnostic work-up or the follow-up study for the presumed or the alleged large-vessel cerebrovascular disease. Among these, 35 patients (29 men, 6 women; mean age, 64.8 \pm 13.2 years; range, $23-81$ years) who had occlusion of unilateral ICA with primary collateral pathways via circle of Willis were identified.

The identified patients were classified into three groups according to their clinical features. First group was composed of asymptomatic patients without any cerebral infarction (group 1). Second group consisted with stable patients with chronic infarction, which was present in the ipsilateral cerebral hemisphere to the occluded ICA (group 2). We defined stable clinical status as follows: a) no newly developed or progressed neurologic deficit for at least 6 months before the performance of Q-MRA, and b) no radiologic evidence of newly developed ischemic lesion on brain imaging. Symptomatic patients with ipsilateral acute/subacute infarction were grouped into the third group (group 3): accordingly, these patients had acute ischemic event within 1 month before Q-MRA. During this process, 2 patients in the second group were excluded for the reasons detailed here: one patient was diagnosed with Takayasu arteritis later, and the other patient showed progression of neurologic symptom regardless of the development of new infarction. Regarding the latter patient, the reason of the patient's symptom progression was not clear.

Clinical information was obtained by reviewing the electronic medical records. Demographic data including age, sex, and medical history was acquired. The institutional review board approved this study. Informed consent requirements were waived by the board.

\section{Imaging protocol and analysis}

The protocol of Q-MRA was detailed in a previous study. ${ }^{11}$ Q-MRA was performed with on 3T MRI scanner (Achieva, Philips Healthcare, Best, the Netherlands), and the mVFR was calculated from NOVA software on a separate workstation.

A standard axial 3-dimensional time-of-flight (TOF) MRA of intracranial and cervical arteries was obtained (Figure 1A). Then, the acquired images were transmitted to the workstation to reconstruct 3D surface-rendered vessel images (Figure 1B). ${ }^{12}$ After determining optimal perpendicular scan plane and setting the baseline coordinates following the method from the previous study, ${ }^{12}$ retrospectively gated, fast 2 -dimensional phase-contrast sequence was performed using the parameters as follows: repetition time, 10-15 ms; echo time, $407 \mathrm{~ms}$; flip angle, $15^{\circ}$; number of excitation, 1; field-of-view, $250 \times 250 \mathrm{~mm}^{2}$; acquisition matrix, $256 \times 128 \mathrm{~mm}^{2}$; slice thickness, $4 \mathrm{~mm}$ for intracranial arteries and $5 \mathrm{~mm}$ for cervical arteries. Velocity encoding was automatically adjusted by NOVA software.

For the calculation of mVFR, the obtained phase-contrast images were transmitted to the workstation. A region-of-interest was automatically located on the acquired phase-contrast images and displayed on the 3D surface-rendered images for the vessel verification. The mVFR for each intracranial artery was calculated in milliliters per minute $(\mathrm{mL} / \mathrm{min})$, and reported (Figure 1C). The total scan time for the entire study was 20-30 minutes.

In addition, the following sequences were obtained according to the clinical need: diffusion-weighted imaging, T1- and T2weighted turbo spin-echo imaging, Fluid-attenuated inversion recovery imaging, gradient recoiled echo $\mathrm{T} 2{ }^{*}$-weighed imaging, dynamic susceptibility perfusion magnetic resonance imaging (MRI), gadolinium-enhanced T1-weighted imaging, and/or contrast-enhanced MRA.

The degree of contralateral ICA stenosis was assessed according to the following criteria: mild, $0 \%-49 \%$; moderate, $50 \%$ $69 \%$; severe, $70 \%-99 \%$; occlusion, $100 \%$. To indirectly assess 

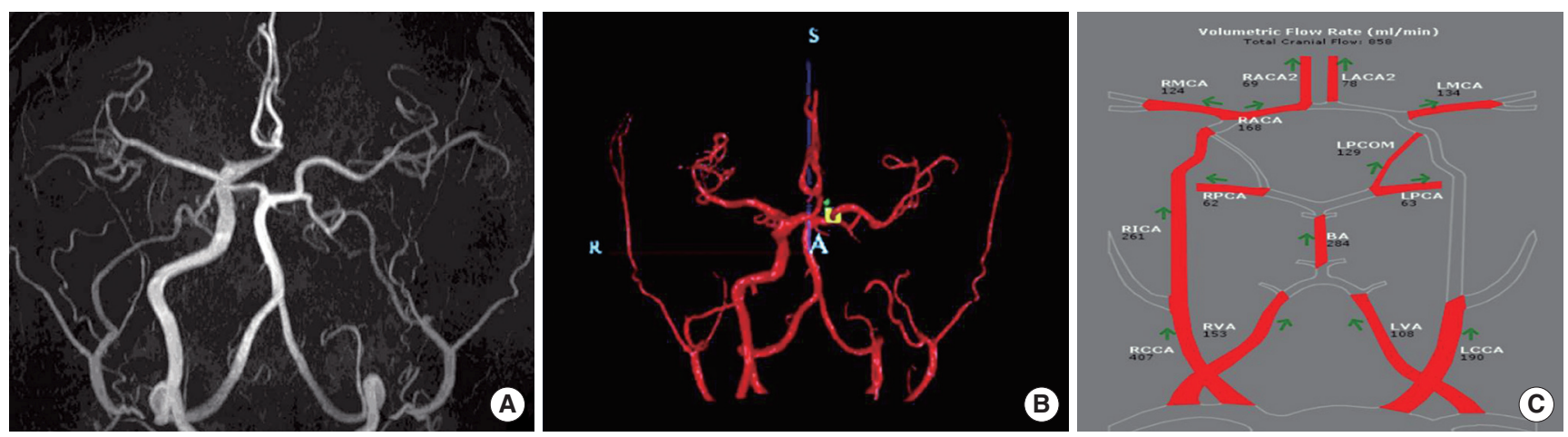

Figure 1. Q-MRA images of a 47-year-old male patient in group 1. (A) Left ICA is occluded, but patent ipsilateral MCA flow from posterior communicating arterial collateral is demonstrated on TOF-MRA. (B) The section plane (yellow) shows the location of the measurement of left MCA flow. (C) The overall mVFR of the intracranial and cervical arteries are shown in the report of NOVA software.

the volume of the infarction, MRI Alberta Stroke Program Early CT score (ASPECTS) was calculated for each patient. ${ }^{13}$

\section{mVFR calculation}

The mVFR was calculated at anterior cerebral artery (ACA), MCA and posterior cerebral artery (PCA) in both ipsilateral and contralateral sides to the occluded ICA. Then, we calculated total ipsilateral and total contralateral cerebral circulation flow by adding up the mVFR from ACA, MCA, and PCA of the same side. We also calculated the ratio of the mVFR of ipsilateral MCA to that of contralateral side, and that of total ipsilateral cerebral circulation flow to contralateral side, respectively.

\section{Statistical analysis}

Categorical variables are expressed as frequencies with percentages. Continuous variables are presented as mean values \pm standard deviations. Demographic data between the patients' groups were compared using Kruskal-Wallis tests and Fisher's exact tests. MRI ASPECTS between the group of stable patients with chronic infarction and that of symptomatic patients with acute/subacute infarction was compared using Mann-Whitney test. Kruskal-Wallis test was also used to compare the calculated values of mVFR between 3 patients' groups. If needed, further subgroup analysis was additionally performed. A $P$ value less than 0.05 was considered statistically significant. The statistical analyses were performed using a statistical software package (SPSS, version 17.0; SPSS, Chicago, IL, USA).

\section{Results}

\section{Subjects}

As a result, 6 patients were classified into group 1, 15 patients into group 2 , and 12 patients into group 3 . As for the group 3, 8 patients had hemodynamic dominant infarction (watershed
Table 1. Demographic and clinical findings of the subjects with unilateral internal carotid artery (ICA) occlusion

\begin{tabular}{|c|c|c|c|c|}
\hline & $\begin{array}{c}\text { Group 1* } \\
(n=6)\end{array}$ & $\begin{array}{l}\text { Group } 2^{\dagger} \\
(n=15)\end{array}$ & $\begin{array}{l}\text { Group } 3^{\ddagger} \\
(n=12)\end{array}$ & $P$ value $e^{\S}$ \\
\hline Men/women, n (\%) & $6 / 0(100)$ & 13/2 (86.7) & $8 / 4(66.7)$ & 0.18 \\
\hline $\begin{array}{l}\text { Age, mean } \pm \text { standard } \\
\text { deviation (yr) }\end{array}$ & $63.5 \pm 12.7$ & $65.3 \pm 13.8$ & $65.8 \pm 11.4$ & 0.9 \\
\hline Hypertension, $\mathrm{n}(\%)$ & $2(40)$ & $8(53.3)$ & $9(75)$ & 0.22 \\
\hline Diabetes mellitus, n (\%) & $0(0)$ & $5(33.3)$ & $6(50)$ & 0.11 \\
\hline Heart disease, $\mathrm{n}(\%)$ & $3(50)$ & $2(13.3)$ & $0(0)$ & 0.12 \\
\hline Current smoking, n (\%) & $1(20)$ & $3(20)$ & $4(33.3)$ & 0.65 \\
\hline Hyperlipidemia, n (\%) & $3(60)$ & $6(40)$ & $5(41.7)$ & 0.91 \\
\hline \multicolumn{5}{|l|}{ Degree of contralateral } \\
\hline \multicolumn{5}{|l|}{ ICA stenosis, n (\%) } \\
\hline $0 \%-49 \%$ & $6(100)$ & $14(93.3)$ & $12(100)$ & 0.54 \\
\hline $50 \%-69 \%$ & $0(0)$ & $0(0)$ & $0(0)$ & - \\
\hline $70 \%-99 \%$ & $0(0)$ & $1(6.7)$ & $0(0)$ & 0.54 \\
\hline $100 \%$ & $0(0)$ & $0(0)$ & $0(0)$ & - \\
\hline $\begin{array}{l}\text { Alberta Stroke Program } \\
\text { Early CT score (mean } \pm \text { SD) }\end{array}$ & - & $8.67 \pm 1.91$ & $8.33 \pm 0.98$ & 0.11 \\
\hline
\end{tabular}

*Group1, asymmetric patients; ${ }^{\dagger}$ Group 2, stable patients with chronic infarction; ${ }^{\ddagger}$ Group 3, symptomatic patients with acute/subacute infarction; ${ }^{\S} P$ value is calculated by Kruskal-Wallis test and Mann-Whitney test.

zone infarction), and 4 patients had non-hemodynamic dominant infarction such as embolic infarction. Since we initially included patients with patent primary collateral pathways via anterior communicating and/or posterior communicating arterial flows, there was no significant difference of collateral pathways between the patients' groups.

Table 1 demonstrates the patients' clinical characteristics, degree of contralateral ICA stenosis, and MRI ASPECTS. There was no significant difference in the patients' age, sex, and medical history related to the vascular ischemic risk factor. No patient had occlusion of contralateral ICA. MRI ASPECTS between the stable patients with chronic infarction and the symptomatic patients with acute/subacute infarction was not significantly different from each other. 
Table 2. Flow quantification of intracranial arteries using quantitative magnetic resonance angiography (Q-MRA)

\begin{tabular}{|c|c|c|c|c|}
\hline & $\begin{array}{c}\text { Group } 1 \\
(n=6)\end{array}$ & $\begin{array}{l}\text { Group } 2 \\
(n=15)\end{array}$ & $\begin{array}{l}\text { Group } 3 \\
(n=12)\end{array}$ & $P$ value \\
\hline \multicolumn{5}{|c|}{ Mean volume flow rate (mVFR), mean \pm standard deviation (mL/min) } \\
\hline Ipsilateral anterior cerebral artery (ACA) & $75.0 \pm 28.7$ & $57.3 \pm 24.3$ & $67.5 \pm 33.3$ & 0.49 \\
\hline Contralateral ACA & $73.7 \pm 33.4$ & $65.3 \pm 27.4$ & $58.0 \pm 12.4$ & 0.21 \\
\hline Ipsilateral middle cerebral artery (MCA) & $121.8 \pm 42.0$ & $119.9 \pm 36.1$ & $73.7 \pm 45.6$ & $0.04^{*}$ \\
\hline Contralateral MCA & $167.3 \pm 45.0$ & $163.3 \pm 50.5$ & $170.2 \pm 45.5$ & 0.9 \\
\hline Ipsilateral posterior cerebral artery (PCA) & $117.2 \pm 48.9$ & $104.8 \pm 38.7$ & $88.2 \pm 49.9$ & 0.48 \\
\hline Contralateral PCA & $79.0 \pm 14.6$ & $77.1 \pm 26.0$ & $68.1 \pm 29.8$ & 0.59 \\
\hline Contralateral internal carotid artery (ICA) & $350.0 \pm 112.6$ & $360.8 \pm 85.8$ & $349.5 \pm 110.6$ & 0.82 \\
\hline Ipsilateral ACA+MCA+PCA & $314.0 \pm 44.4$ & $282.0 \pm 68.6$ & $229.3 \pm 51.3$ & $0.02^{*}$ \\
\hline \multicolumn{5}{|l|}{ The ratio of mVFR (\%) } \\
\hline $\begin{array}{l}\text { Ipsilateral to contralateral MCA } \\
\text { Ipsilateral to contralateral }\end{array}$ & $74.8 \pm 24.3$ & $75.7 \pm 18.0$ & $46.2 \pm 26.8$ & $0.02^{*}$ \\
\hline $\mathrm{ACA}+\mathrm{MCA}+\mathrm{PCA}$ & $99.7 \pm 11.5$ & $97.5 \pm 35.8$ & $79.6 \pm 16.6$ & $0.04^{*}$ \\
\hline
\end{tabular}

Group1, asymptomatic patients; Group 2, stable patients with chronic infarction; Group 3; symptomatic patients with acute/subacute infarction. Pvalue is calculated by Kruskal-Wallis test.

${ }^{*}$ Pvalue less than 0.05 .

\section{Quantitative mVFR}

Table 2 summarizes the results of the quantitative mVFR in intracranial arteries. The mVFR of MCA ipsilateral to the occlusion of ICA was significantly lower in the symptomatic patients with acute/subacute infarction than those in the stable patients with chronic infarction or the asymptomatic patients (group 1, $121.8 \pm 42.0 \mathrm{~mL} / \mathrm{min}$; group $2,119.9 \pm 36.1 \mathrm{~mL} / \mathrm{min}$; group 3 , $73.7 \pm 45.6 \mathrm{~mL} / \mathrm{min} ; P=0.04)$. Total ipsilateral cerebral circulation flow - the sum of mVFR from ipsilateral ACA, MCA, and PCA - was also significantly lower in the acute symptomatic patients than those in other groups (group 1, 314.0 $\pm 44.4 \mathrm{~mL} /$ $\mathrm{min}$; group 2, $282.0 \pm 68.6 \mathrm{~mL} / \mathrm{min}$; group 3, $229.3 \pm 51.3 \mathrm{~mL} /$ $\min ; P=0.02$ ). There was no significant difference in above two values between the stable patients with chronic infarction and the asymptomatic patients.

The ratio of mVFR of ipsilateral MCA to that of contralateral side was significantly different between the patients' groups (group 1, $74.8 \pm 24.3 \%$; group 2, $75.7 \pm 18.0 \%$; $46.2 \pm 26.8 \%$; $P=0.02)$. In subgroup analysis, the value was significantly lower in symptomatic patients with acute/subacute infarction than in stable patients with chronic infarction $(P<0.01)$. The value in symptomatic patients was also lower than asymptomatic patients, but did not reach the statistical significance $(P=0.07)$.

The ratio of total ipsilateral cerebral circulation flow to total contralateral cerebral circulation flow was significantly different between the patients' groups (group 1, 99.7 $\pm 11.5 \%$; group 2, $97.5 \pm 35.8 \%$; group $3,79.6 \pm 16.6 \%$; $P=0.04$ ). In subgroup analysis, the difference between group 1 and 3 was statistically significant $(P=0.01)$. The value from group 3 was lower than that from group 2 , but it did not reach the statistical significance $(P=0.09)$.

\section{Representative Cases}

Figure 2 presents the representative images from two patients from group 2 and group 3, respectively. A 67-year-old male in group 2 showed about $31 \mathrm{~mm}$ sized chronic infarction with tissue loss in right corona radiata (Figure $2 \mathrm{~A}$ ). He showed no neurologic symptom relevant to this lesion at the time of imaging. The other patient in group 3 was an 80-year-old male who visited the hospital with sudden onset right hand weakness. On diffusion-weighted imaging, about $33 \mathrm{~mm}$ sized acute infarction was noted in left corona radiata (Figure 2B). On TOF MRA, patent ipsilateral primary collateral flow via circle of Willis was equally verified in both patients (Figure $2 \mathrm{C}$ and $\mathrm{D}$ ). However, when we performed Q-MRA and measured collateral flow in intracranial arteries, the mVFR of ipsilateral MCA $(94 \mathrm{~mL} / \mathrm{min}$ vs. $65 \mathrm{~mL} / \mathrm{min})$, the $\mathrm{mVFR}$ of total ipsilateral cerebral circulation $(244 \mathrm{~mL} / \mathrm{min}$ vs. $199 \mathrm{~mL} / \mathrm{min})$, the ratio of $\mathrm{mVFR}$ of ipsilateral MCA to that of contralateral side (95.9\% vs. $50.0 \%$ ), and the ratio of total ipsilateral cerebral circulation flow to total contralateral cerebral circulation flow (102.5\% vs. $89.2 \%$ ) was all lower in the patient from group 3 than the patient from group 2.

\section{Discussion}

The degree of intracranial collateral flows has been mainly evaluated using regional cerebral blood flow, which can be assessed by diverse modalities including technetium-99m-hexamethyl propyleneamineoxime single-photon emission computed tomography, ${ }^{14,15}$ perfusion computed tomography, ${ }^{16,17}$ and perfusion MRI such as dynamic susceptibility perfusion $\mathrm{MRI}^{18}$ or arterial spin-labeling perfusion MRI. ${ }^{19}$ Although cerebral blood flow may provide information about tissue-level collater- 

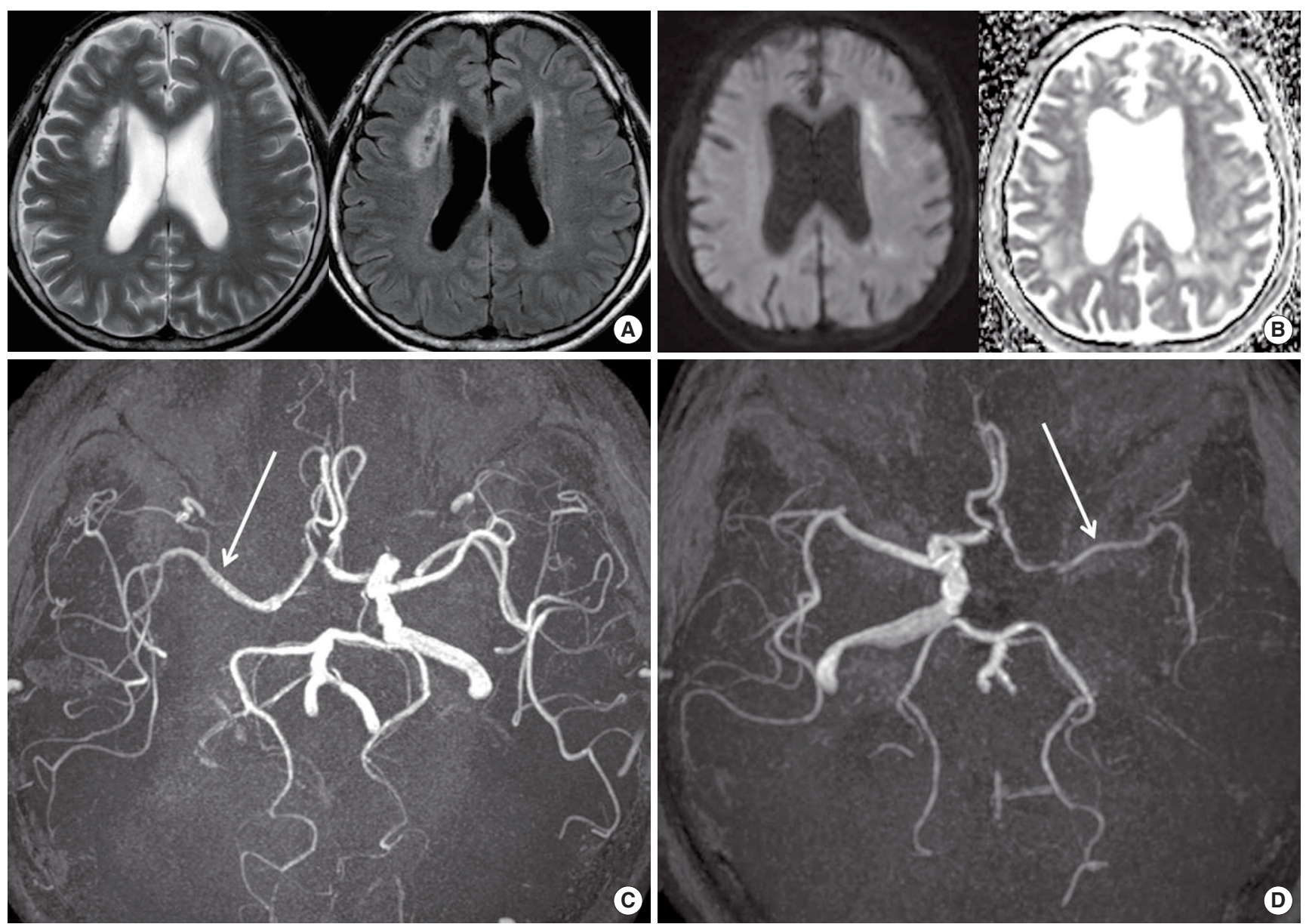

Figure 2. (A) T2-weighted imaging and fluid-attenuated inversion recovery images of a 67-year-old male patient. About $3.1 \mathrm{~cm}$ sized chronic infarction is noted in right anterior corona radiata. (B) Diffusion-weighted imaging and Apparent diffusion coefficient map of an 80-year-old male. He had sudden onset right hand weakness at the time of imaging. There is about $3.3 \mathrm{~cm}$ sized acute infarction in left anterior corona radiata. (C) TOF MRA of the 67-year-old male with chronic infarction. Patent flow of MCA ipsilateral to the occluded ICA is visualized. The mVFR of the ipsilateral MCA was calculated as $94 \mathrm{~mL} / \mathrm{min}$. (D) The 80-year-old male with acute infarction in the territory in left MCA also shows intact flow of ipsilateral MCA to the occluded ICA on TOF MRA. However, the mVFR of this MCA was calculated as $65 \mathrm{~mL} / \mathrm{min}$.

al flow adequacy, it is incapable of quantitatively demonstrating collateral flow routes. ${ }^{8}$

The direct measurement of volume flow via collateral pathways or intracranial arteries has not been commonly performed. A few studies in late 1900's used transcranial Doppler to evaluate the velocity of ipsilateral MCA via primary collateral pathways in ICA occlusion. ${ }^{20,21}$ These studies reported that diminished blood flow velocity less than approximate $30 \mathrm{~cm} / \mathrm{sec}$ was associated with poor clinical prognosis. ${ }^{20,21}$ However, transcranial Doppler is an operator-dependent, subjective study which cannot provide objective result of volume flow measurement. ${ }^{8}$

Recently, the direct vascular flow measurement has become possible with the use of Q-MRA. ${ }^{7}$ Q-MRA is a technique that uses TOF and phase-contrast MRI to visualize extracranial and intracranial vascular anatomy, and provide the direct measurement of volumetric blood flow within intracranial arteries. ${ }^{7,22}$ The accuracy of the flow measurement using Q-MRA has been extensively tested with phantom blood flow simulators. ${ }^{11,23}$ Many previous studies also have verified the reproducibility of flow measurement using Q-MRA, and intra-rater and inter-rater reliabilities of Q-MRA were also investigated. ${ }^{11,2426}$ In addition, the flow measurement from Q-MRA has proved to be correlated with several other methods such as $\mathrm{H} 2(15) \mathrm{O}$ positron emission tomography, ${ }^{26}$ ultrasound vector velocity technique, ${ }^{27}$ laser Doppler velocimetry and computational fluid dynamics. ${ }^{28}$

Our study showed that Q-MRA using NOVA software could be a feasible tool for the direct measurement of the volume flow of intracranial arteries including primary collateral flow via circle of Willis in ICA occlusion. Moreover, we demonstrated that the mVFR of ipsilateral MCA and that of total ipsilateral cerebral circulation were reduced in the symptomatic patients with acute/ subacute infarction than in the stable patients with chronic infarction or in the asymptomatic patients without any infarction. The ratio of mVFR in ipsilateral to contralateral MCA and that 
of total mVFR in ipsilateral cerebral circulation to contralateral side also decreased in the symptomatic group.

It has been well known that reduced primary Willisian collateral flow is related to the development of cerebral ischemia. ${ }^{5,10}$ The North American Symptomatic Carotid Endarterectomy Trial study proved that increased collateral flow via anterior communicating artery or posterior communicating artery was associated with lower cerebral ischemic risks. ${ }^{4}$ Our study has its merit in that, with the use of Q-MRA, we objectively verified the larger reduction in the amount of primary Willisian collateral flow in the symptomatic patients with ICA occlusion and ipsilateral recent infarction than in the neurologically stable or asymptomatic patients.

Our study has some limitations. First, this study was crosssectional study with small number of patients. In this regard, there could be selection bias from small size of study population, and age correction of mVFR could not be achieved. However, the statistical analysis verified that the included patients showed similar clinical risk factors for stroke, which could give credibility to the comparison of mVFR between the patients' groups. Still, to determine whether decreased collateral flow can really predict the future development of symptomatic ischemia in ICA occlusion, further follow-up prospective study with larger number of patients will be necessary. Second, we did not directly measure the volume of the infarcted cerebral tissue in the assessment of the collateral degree. It can be easily anticipated that the degree of collateral flow will be measured low in the larger infarcted area than in the smaller one. However, we compared the values of MRI ASPECTS between the symptomatic patients with acute/subacute infarction and the stable patients with chronic infarction, which could reflect the volume of the infarcted area. ${ }^{29}$ Therefore, the difference in the infarction volume would have had little impact on the result of our study.

\section{Conclusions}

In conclusion, Q-MRA can demonstrate the difference in the degree of primary collateral flow via circle of Willis in unilateral ICA occlusion. The volume flow in MCA and in total cerebral circulation was significantly reduced in the patients with acute/ subacute symptomatic ipsilateral cerebral infarction than in the patients without. Further follow-up study with prospective nature and the larger number of patients will be needed to solidify the result from this study.

\section{References}

1. Kang J, Kim N, Oh CW, Kwon OK, Jung CK, Kim WJ, et al.
Symptomatic steno-occlusion of cerebral arteries and subsequent ischemic events in patients with acute ischemic stroke. $J$ Stroke Cerebrovasc Dis 2014;23:e347-e353.

2. Liebeskind DS, Flint AC, Budzik RF, Xiang B, Smith WS, Duckwiler GR, et al. Carotid I's, L's and T's: collaterals shape the outcome of intracranial carotid occlusion in acute ischemic stroke. J Neurointerv Surg 2015;7:402-407.

3. Liebeskind DS. Collateral circulation. Stroke 2003;34:22792284.

4. Henderson RD, Eliasziw M, Fox AJ, Rothwell PM, Barnett HJ. Angiographically defined collateral circulation and risk of stroke in patients with severe carotid artery stenosis. North American Symptomatic Carotid Endarterectomy Trial (NASCET) Group. Stroke 2000;31:128-132.

5. Miralles M, Dolz J, Cotillas J, Aldoma J, Santiso MA, Giménez A, et al. The role of the circle of Willis in carotid occlusion: assessment with phase contrast MR angiography and transcranial duplex. Eur J Vasc Endovasc Surg 1995;10:424-430.

6. Kim JT, Park MS, Choi KH, Nam TS, Choi SM, Cho KH. Clinical implications of collateral middle cerebral artery flow in acute ischaemic stroke with internal carotid artery occlusion. Eur J Neurol 2011;18:1384-1390.

7. Conway SA, Bowling SM, Geyer JD, Potts DC, Clemons BL, Gomez CR. Quantitative magnetic resonance angiography of the cerebrovasculature in physiologic and pathologic states. $J$ Neuroimaging 2008; 18:34-37.

8. Ruland S, Ahmed A, Thomas K, Zhao M, Amin-Hanjani S, Du $\mathrm{X}$. Leptomeningeal collateral volume flow assessed by quantitative magnetic resonance angiography in large-vessel cerebrovascular disease. J Neuroimaging 2009; 19:27-30.

9. Rutgers D, Klijn C, Kappelle L, van der Grond J. Recurrent stroke in patients with symptomatic carotid artery occlusion is associated with high-volume flow to the brain and increased collateral circulation. Stroke 2004;35:1345-1349.

10. Kluytmans M, Van der Grond J, Van Everdingen K, Klijn CJ, Kappelle LJ, Viergever MA. Cerebral hemodynamics in relation to patterns of collateral flow. Stroke 1999;30:1432-1439.

11. Zhao M, Amin-Hanjani S, Ruland S, Curcio AP, Ostergren L, Charbel FT. Regional cerebral blood flow using quantitative MR angiography. AJNR Am J Neuroradiol 2007;28:1470-1473.

12. Zhao M, Charbel FT, Alperin N, Loth F, Clark ME. Improved phase-contrast flow quantification by three-dimensional vessel localization. Magn Reson Imaging 2000;186:697-706.

13. Barber P, Hill M, Eliasziw M, Demchuk AM, Pexman JH, Hudon ME, et al. Imaging of the brain in acute ischaemic stroke: comparison of computed tomography and magnetic resonance diffusion-weighted imaging. J Neurol Neurosurg Psychiatry 2005;76:1528-1533. 
14. Palestro CJ, Sen C, Muzinic M, Afriyie M, Goldsmith SJ. Assessing collateral cerebral perfusion with technetium-99mHMPAO SPECT during temporary internal carotid artery occlusion. J Nucl Med 1993;34:1235-1238.

15. Tanaka F, Nishizawa S, Yonekura Y, Sadato N, Ishizu K, Okazawa $\mathrm{H}$, et al. Changes in cerebral blood flow induced by balloon test occlusion of the internal carotid artery under hypotension. Eur J Nucl Med 1995;22:1268-1273.

16. Gibbs J, Leenders K, Wise R, Jones T. Evaluation of cerebral perfusion reserve in patients with carotid-artery occlusion. Lancet 1984;323:310-314.

17. Wintermark M, Reichhart M, Thiran JP, Maeder P, Chalaron M, Schnyder P, et al. Prognostic accuracy of cerebral blood flow measurement by perfusion computed tomography, at the time of emergency room admission, in acute stroke patients. Ann Neurol 2002;51:417-432.

18. Teng MM, Cheng HC, Kao YH, Hsu LC, Yeh TC, Hung CS, et al. MR perfusion studies of brain for patients with unilateral carotid stenosis or occlusion: evaluation of maps of "time to peak" and "percentage of baseline at peak". J Comput Assist Tomogr 2001;25:121-125.

19. Hendrikse J, van Osch MJ, Rutgers DR, Bakker CJ, Kappelle LJ, Golay X, et al. Internal carotid artery occlusion assessed at pulsed arterial spin-labeling perfusion MR imaging at multiple delay times. Radiology 2004;233:899-904.

20. Halsey J Jr. Prognosis of acute hemiplegia estimated by transcranial Doppler ultrasonography. Stroke 1988;19:648-649.

21. Hedera P, Traubner P, Bujdáková J. Short-term prognosis of stroke due to occlusion of internal carotid artery based on transcranial Doppler ultrasonography. Stroke 1992;23:1069-1072.

22. Brisman JL, Pile-Spellman J, Konstas AA. Clinical utility of quantitative magnetic resonance angiography in the assess- ment of the underlying pathophysiology in a variety of cerebrovascular disorders. Eur J Radiol 2012;81:298-302.

23. Zhao M, Curcio AP, Clark ME, Zhou X. In vitro validation of MR volumetric flow measurement. Proceedings of the 2004 International Workshop on Flow and Motion 2004:148-149.

24. Ruland S, Zhao M, Pandey D, Ostergren L, Amin-HanjaniS, Curcio A, et al. Reproducibility of cerebral blood flow analysis using quantitative magnetic resonance angiography. Presented at the AANS/CNS Cerebrovascular Section 9th Joint Annual Meeting February 17-20, 2006, Orlando, FL.

25. Zhao M. Repeatability of MR volumetric flow measurements in major cerebral arteries. Presented at 2006 ISMRM Flow and Motion Study Group Workshop: Imaging Assessment of Cardiovascular and Tissue Mechanics July 13-16, 2006, New York, NY.

26. Carroll TJ, Teneggi V, Jobin M, Squassante L, Treyer V, Hany TF, et al. Absolute quantification of cerebral blood flow with magnetic resonance, reproducibility of the method, and comparison with $\mathrm{H} 2$ (15)O positron emission tomography. J Cereb Blood Flow Metab 2002;22:1149-1156.

27. Hansen KL, Udesen J, Oddershede N, Henze L, Thomsen C, Jensen JA, et al. In vivo comparison of three ultrasound vector velocity techniques to MR phase contrast angiography. Ultrasonics 2009;49:659-667.

28. Hollnagel DI, Summers PE, Poulikakos D, Kollias SS. Comparative velocity investigations in cerebral arteries and aneurysms: 3D phase-contrast MR angiography, laser Doppler velocimetry and computational fluid dynamics. NMR Biomed 2009;22:795-808.

29. Lin K, Lee SA, Zink WE. What ASPECTS value best predicts the $100-\mathrm{mL}$ threshold on diffusion weighted imaging? Study of 150 patients with middle cerebral artery stroke. J Neuroimaging 2011;21:229-231. 